\title{
Clinical study of benign and malignant fibrous-osseous lesions of the jaws
}

\author{
Ju-Min Lee, Won-Wook Song, Jae-Yeoul Lee, Dae-Seok Hwang, \\ Yong-Deok Kim, Sang-Hun Shin, In-Kyo Chung, Uk-Kyu Kim \\ Department of Oral and Maxillofacial Surgery, School of Dentistry, Pusan National University, Busan, Korea
}

Abstract (J Korean Assoc Oral Maxillofac Surg 2012;38:29-37)

Introduction: Fibrous-osseous lesions of the jaws are difficult to diagnose precisely until excised biopsy results are found, so they might be confused with malignant lesions. This clinical study focused on the diagnostic aids of lesions that demonstrate different clinical, radiologic, and histological findings.

Materials and Methods: A total of 16 patients with benign fibrous-osseous lesions on the jaws (6 fibrous dysplasias, 6 ossifying fibromas, 3 cemental dysplasias, and one osteoblastoma) were reviewed. Nine patients with malignant fibrous-osseous lesions (8 osteosarcomas and one Ewing's sarcoma) were also retrospectively reviewed.

Results: Osteosarcoma patients complained of facial swelling and tooth mobility. The radiographic findings showed the irregular resorption of cortical bone and periosteal reactions. Histological features included cellular pleomorphism and atypical mitosis. An Ewing's sarcoma patient complained of tooth mobility and facial swelling. Onion-skin appearance and irregular expansile marginal bony radiolucency were seen in the radiography. Fibrous dysplasia patients complained of facial swelling and asymmetry. The radiographic features were mostly ground-glass radiopacity. Histological findings showed a bony trabeculae pattern surrounded by fibrous ground substances. Ossifying fibroma patients complained of buccal swelling and jaw pains, showing expanded cortical radiolucent lesions with a radiopaque margin. Histological findings were revealed as cellular fibrous stroma with immature woven bones. In cemental dysplasia, most of their lesions were found in a routine dental exam. Well-circumscribed radiopaque lesions were observed in the radiography, and cementum-like ossicles with fibrous stroma were seen in the microscopy. An osteoblastoma patient complained of jaw pain and facial swelling. Radiographic findings were mottled, dense radiopacity with osteolytic margin. Trabeculae of the osteoid with a vascular network and numerous osteoblasts with woven bone were predominantly found in the microscopy.

Conclusion: Our study showed similar results as other studies. We suggest the clinical parameters of diagnosis and treatment for malignant and benign fibrous-osseous lesions of the jaws.

Key words: Fibrous-osseous lesions of the jaws, Fibrous dysplasia, Ossifying Fibroma, Ewing's sarcoma, Osteoblastoma

[paper submitted 2011. 8. 25 / revised 2011. 11. 15 / accepted 2012. 1. 20]

\section{Introduction}

Many scholars have been conducted studies on fibrousosseous lesions occurring in the facial bone since the systematized report of von Recklinghausen ${ }^{1}$ around 1891. The manifestation pattern and radiographic and histological

\section{Uk-Kyu Kim}

Department of Oral and Maxillofacial Surgery, School of Dentistry, Pusan National University, Beomeo-ri, Mulgeum-eup, Yangsan 626-770, Korea TEL: +82-55-360-5103 FAX: +82-55-360-5104

E-mail: kuksjs@pusan.ac.kr

(c) This is an open-access article distributed under the terms of the Creative Commons Attribution Non-Commercial License (http://creativecommons.org/licenses/by-nc/3.0/), which permits unrestricted non-commercial use, distribution, and reproduction in any medium, provided the original work is properly cited. features of each disease were similar, and some scholars tried to classify the fibro-osseous lesions according to the etiology and conducted studies on whether the cemental lesion originates from the periodontal ligament or endosteal cells $\mathrm{s}^{2-5}$. Fibrous-osseous lesions are mostly composed of benign tumors such as fibrous dysplasia, ossifying fibroma, cemental dysplasia and osteoblastoma. However, malignant tumors such as osteosarcoma ${ }^{6,7}$ and Ewing's sarcoma ${ }^{8}$ also exists. Therefore, when the fibrous-osseous lesion patient comes to the hospital, oral and maxillofacial surgeons should diagnose differentially whether the lesion is malignant or benign. Through our clinical study, we are willing to find out a diagnostic parameter for each benign and malignant lesion by analyzing the differences on diagnostic criteria, radiographic

*This study was conducted with the support of research fund by Pusan National University in 2011. 
findings, histological feateures and the prognosis lesions retrospectively.

\section{Materials and Methods}

We analyzed the clinical data, radiographs, and histopathologic specimens of the patients who were diagnosed as malignant and benign fibrous-osseous lesions on the jaws in the department of oral and maxillofacial surgery, Pusan National University Hospital from 2004 to 2008. A total of 16 benign fibrous-osseous lesions and 9 malignant fibrousosseous lesions were included. Benign lesions included 6 fibrous dysplasia, 6 ossifying fibroma, 3 cemental dysplasia, and one osteoblastoma, whereas the malignant lesions included 8 osteosarcoma and one Ewing's sarcoma.

\section{Results}

As clinical findings from 8 patients out of a total of 9 patients diagnosed with malignant fibrous-osseous lesion, the chief complaints were facial swelling and tooth mobility that mostly developed on the mandible. One patient died during the period of diagnostic workup before treatment. The sex ratio was almost even, and the age distribution of patients with osteosarcoma ranged from the third to fifth decades. Primarily, wide excision was performed, and some patients received adjuvant chemotherapy. In case of Ewing's sarcoma, chemotherpy was underwent. In the 7 of all cases with osteosarcoma, excision was performed, the survival rates were $63 \%$ (2-year observation period) and $28 \%$ recurred after the surgery.(Table 1)

A total of 16 patients were diagnosed as benign fibrousosseous lesion, and age distribution of patients was from the second to seventh decades with little difference in sex. The chief complaints of the patients were facial swelling with occasional jaw pains, and predilection site was mostly the posterior mandible. The lesions were classified as fibrous dysplasia, ossifying fibroma, cemental dysplasia, and

Table 1. Summary of cases diagnosed as malignant fibrous-osseous lesions

\begin{tabular}{ccllll}
\hline Age (year) & Sex & \multicolumn{1}{c}{ Chief complaint } & Site involved & \multicolumn{1}{c}{ Diagnosis } & \multicolumn{1}{c}{ Treatment } \\
\hline 41 & M & Tooth mobility, swelling, pain & Mn Lt body & OS & Partial mandibulectomy, RND, PMMC flap \\
42 & M & Swelling & Mn Lt angle & OS & CTx, wide excision \\
67 & F & Swelling & Mn symphysis & Osteoblastic OS & Composite resection, SOHND, FFF \\
34 & F & Swelling, tooth mobility & Mn Rt post & OS & Composite resection, SOHND, FFF \\
41 & M & Pain, swelling, tooth mobility & Mn Rt & OS & Wide excision, SOHND, FFF \\
8 & F & Swelling & Mn Lt & OS & CTx, expired \\
33 & M & Swelling & Lt cheek, Mn & OS & Wide excision, SOHND \\
29 & F & Swelling, tooth mobility & Ant Mx & OS & Wide excision \\
18 & F & Swelling, tooth mobility & Mn Lt & Ewing's sarcoma & CTx \\
\hline
\end{tabular}

(OS: osteosarcoma, RND: radical neck dissection, PMMC: pectoralis major myocutaneous, CTx: chemotherapy, SOHND: supraomohyoid neck dissection, FFF: fibular free flap, M: male, F: female, Mn: mandible, Lt: left, Rt: right, Ant: anterior, Mx: maxilla)

Ju-Min Lee et al: Clinical study of benign and malignant fibrous-osseous lesions of the jaws. J Korean Assoc Oral Maxillofac Surg 2012

Table 2. Summary of cases diagnosed as benign fibrous-osseous lesions

\begin{tabular}{|c|c|c|c|c|c|}
\hline Age (year) & Sex & Chief complaint & Site involved & Diagnosis & Treatment \\
\hline 15 & $\mathrm{~F}$ & Facial asymmetry & Mn Lt and Mx zygoma & $\mathrm{FD}$ & Bone recontouring \\
\hline 20 & $\mathrm{~F}$ & Facial swelling & Both Mx and Mn Rt, zygoma & Polyostotic FD & Bone recontouring \\
\hline 32 & $\mathrm{~F}$ & None & Mn Lt post & FD & Excision \\
\hline 31 & $\mathrm{~F}$ & Buccal swelling & Mn Rt body & FD & Bone recontouring \\
\hline 39 & M & None & Mx alveolus, palate & FD & Alveoloplasty \\
\hline 62 & M & Gingival swelling & Mn both post & FOD & Excision \\
\hline 50 & $\mathrm{~F}$ & Facial swelling & Mn Lt body, ramus & $\mathrm{OF}$ & Excision \\
\hline 16 & $\mathrm{~F}$ & None & Mn Rt molar & OF & Excision \\
\hline 43 & $\mathrm{~F}$ & Pain, Swelling & Mn both molars & OF & Excision \\
\hline 40 & M & Facial asymmetry & Mn Lt body & OF & Segmental mandibulectomy \\
\hline 16 & $\mathrm{~F}$ & Buccal swelling & Mn Rt molar & OF & Excision \\
\hline 24 & F & None & Mn Rt molar & OF & Excision \\
\hline 21 & $\mathrm{~F}$ & Cancer phobia & Mn Rt premolar & OF & Exploratory biopsy \\
\hline 54 & M & Pain & Mn Rt 3rd molar & OF & Excision \\
\hline 45 & $\mathrm{~F}$ & Pain & Mn Rt molar & $\mathrm{OF}$ & Excision \\
\hline 21 & $\mathrm{~F}$ & Pain, Swelling & Mn Lt molar & Osteoblastoma & Segmental mandibulectomy \\
\hline
\end{tabular}

(FD: fibrous dysplasia, FOD: florid osseous dysplasia, OF: ossifying fibroma, M: male, F: female, Mn: mandible, Lt: left, Rt: right, Mx: maxilla)

Ju-Min Lee et al: Clinical study of benign and malignant fibrous-osseous lesions of the jaws. J Korean Assoc Oral Maxillofac Surg 2012 
osteoblastoma. Lesion was excised and showed no evidence of recurrence.(Table 2)

The radiographic findings from 7 patients with osteosarcoma showed periosteal reaction and pattern of cortical bone destruction. Ewing's sarcoma showed unclear cortical bone margin and onion skin periosteal reaction.

In the histopathological exam, osteosarcoma patients were 5 osteoblastic types and 2 chondroblastic types, and showed pleomorphism of undifferentiated cell and atypical mitosis. Ewing's sarcoma showed vague indistinct cytoplasm and cyto- plasmic membrane and many syncytial-type cells.(Fig. 1)

Patients with fibrous dysplasia had radiological findings of ground-glass feature and histological findings of bony trabeculae with Chinese character. Patients with ossifying fibroma had expansion of cortical bone and resorption of bone margin radiologically. Cellular fibrous stroma and immature woven bone were found with calcified substance. The cemental dysplasia patients showed well-circumscribed radiolucent lesions around mandibular molar teeth radiologically, and cementum-like tissues were seen histologically. Clinically,
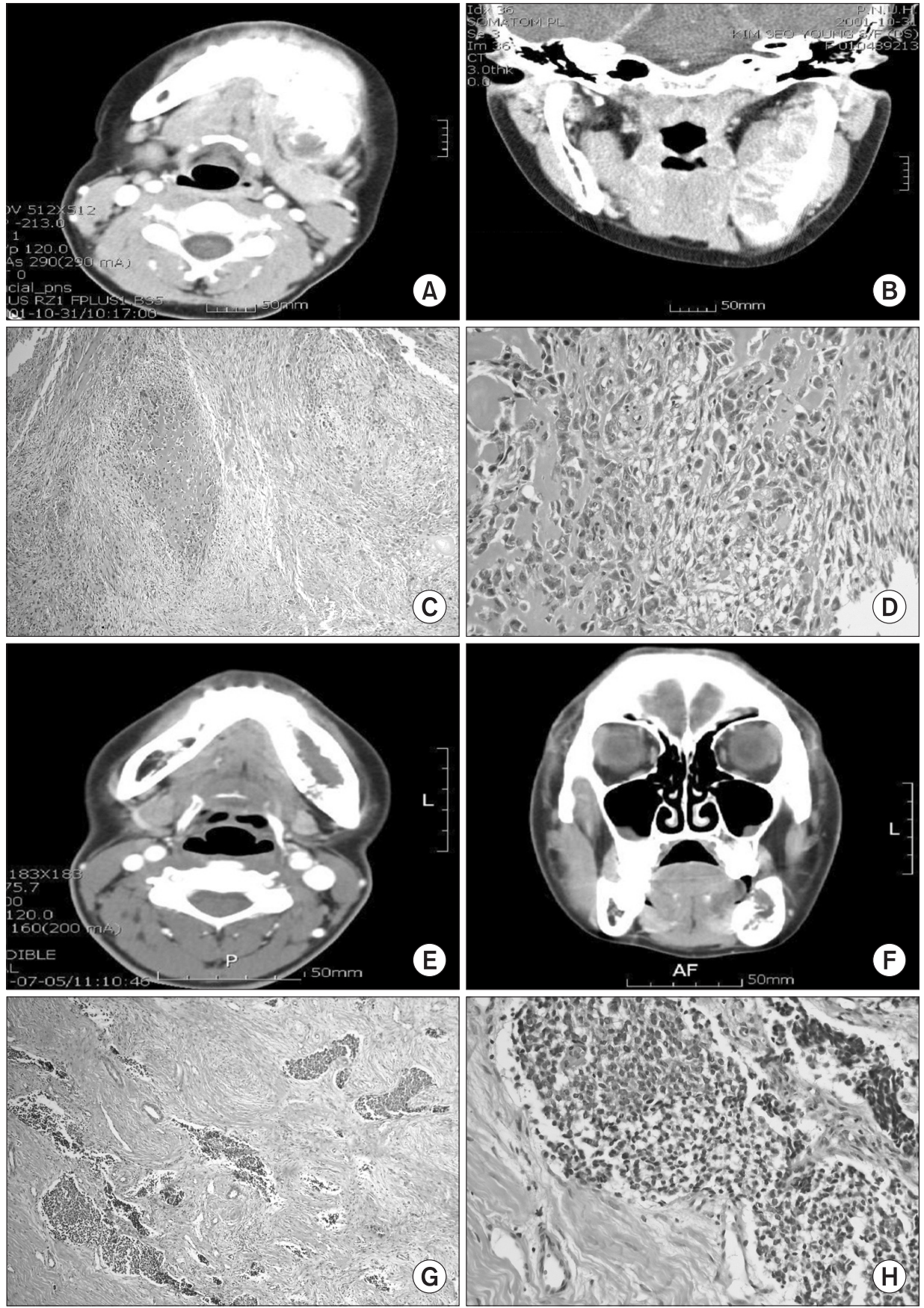

Fig. 1. A-D. Osteosarcoma. E-H. Ewing's sarcoma (H\&E staining, C: $\times 100, \mathrm{D}$ : $\times 200, \mathrm{G}: \times 100, \mathrm{H}: \times 200$ ).

Ju-Min Lee et al: Clinical study of benign and malignant fibrous-osseous lesions of the jaws. J Korean Assoc Oral Maxillofac Surg 2012 

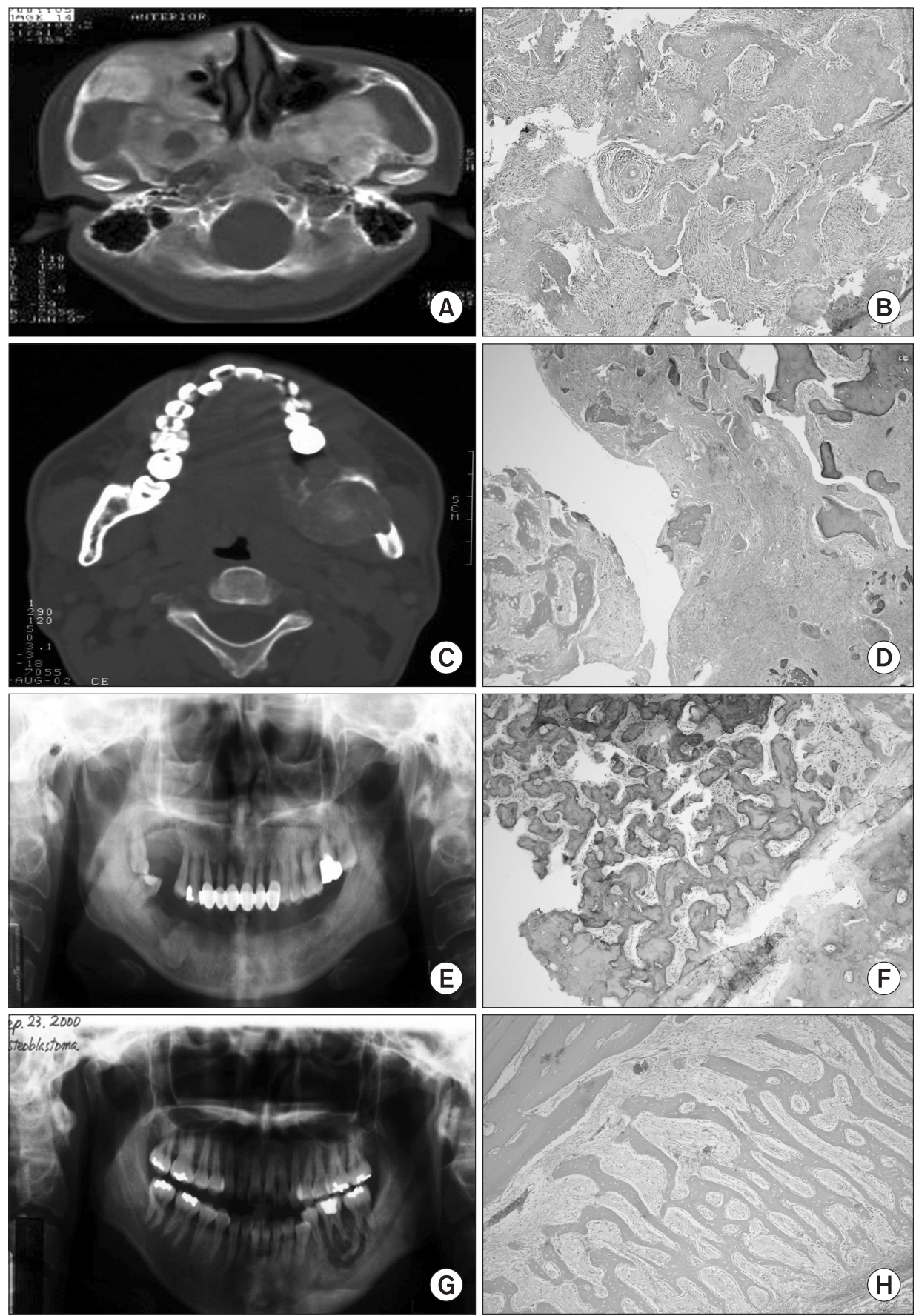

Fig. 2. A, B. Fibrous Dysplasia. C, D. Ossifying Fibroma. E, F. Cemental Dysplasia. G, H. Osteoblastoma (H\&E staining, B: $\times 100, \mathrm{D}: \times 100, \mathrm{~F}: \times 100, \mathrm{H}$ : $\times 100$ ).

Ju-Min Lee et al: Clinical study of benign and malignant fibrous-osseous lesions of the jaws. J Korean Assoc Oral Maxillofac Surg 2012 osteoblastoma seemd to be osteosarcoma, manifested as a mixture of radiolucency and radiopacity. Histologically, bony trabeculae surrounded by vascular network were predominant.(Fig. 2)

\section{Discussion}

Most common malignant fibrous-osseous lesions are osteosarcoma and Ewing's sarcoma ${ }^{6-8}$. Osteosarcoma is a malignant tumor caused by the malignant transformation of mesenchymal cells which have capacity to generate osteoid tissue or immature woven bone. The etiology of this tumor is obscure. Moreover, osteosarcoma in the maxillofacial area occupy only $10 \%$ of total osteosarcoma. The mean ages of onset in maxillofacial area ranges from the 10 s to the $20 \mathrm{~s}$, a little later than those of the other body parts and the survival rate is higher. In this study, the age distribution was 10 s to the 60 s, mostly among over 30s. At least 5 of the $8(63 \%)$ treated cases survived. In cases of osteosarcoma in long bones, a male is prone to the onset than a female; in the head and 
neck, however, the sex ratio is similar. Similar results were deducted in our study. Many other scholars reported a similar onset ratio of osteosarcoma in the maxilla and mandible but this study recorded a ratio of $8: 1$, with higher onset on the mandible. Continuous pain, swelling, and paresthesia have been reported as general symptoms and clinical findings. In our study, facial swelling was a chief complaint, with serous tooth mobility in the lesion. For all patients with osteosarcoma of the jaws, computed tomography (CT) scan and whole body bone scan were performed. A positrom emission tomography-CT scan was utilized when metastasis was doubtful. A CT scan of the head and neck revealed a typical sunburst, sunray appearance caused by the formation of osteophytes in the bone surface of lesion. Such symptom was observed among $60 \%$ of the patients. The periodontal ligament space widening with tooth mobility in panoramic radiographs were observed among $40 \%$ of the patients.

The treatment of osteosarcoma primarily based on wide excision with surgical margin of $3 \mathrm{~cm}$. However surgical margins are often not feasible in head and neck osteosarcoma. In 7 of 8 patients with osteosarcoma except Ewing's sarcoma, excision was performed, and 2 of 7 patients (28\%) recurred. Other treatments in parallel with the surgery for patients with osteosarcoma have been introduced. In case of osteosarcoma in the long bones, chemotherapy with various combination of drugs are used. Chemotherapy in the long bones is performed pre and postoperatively, and survival rates have improved from $20 \%$ to as high as $60-70 \%$ since the beginning of the 1980s in the past. In osteosarcoma in the head and neck, however, the advantage of neoadjuvant chemotherapy is still unclear. Some hypothesis exist for this phenomenon. Micrometastasis of osteosarcoma in the long bones under a microscope reaches $80 \%$, whereas that of osteosarcoma in the head and neck records an $18 \%$ distant metastasis rate. Second, the histological malignancy of osteosarcoma in long bone reaches $85 \%$, whereas the one in the head and neck is $56-79 \%$. Third, the response of osteosarcoma in the head and neck to neoadjuvant chemotherapy is below $25 \%$. Among our clinical cases, 5 cases with surgery were osteoblastic type, and 2 cases were chondroblastic type. Adjuvant chemotherapy was performed in 2 cases with a large lesion. Due to the lack of cases, it was difficult to judge the treatment effects (survival rate and recurrence rate) of adjuvant chemotherapy. According to the operators, there are some literatures on radiation therapy after the surgery in the cases of high grade and infeasible surgical margin. In these cases, there were not any radiation after surgery. For 2 years of follow up, 5 of 8 cases survived.

Continuous observations on osteosarcoma and Ewing's sarcoma are very important and should be checked every two months after surgery. Furthermore, distant metastasis should be checked through chest and abdominal CT scans every 3 months for 2 years, and bone scan using radioactive isotope should be done every 6 months for 2 years; after 2 years, clinical examination and chest $\mathrm{CT}$ scan should be done every year.

Unlike the prognosis of malignant fibrous-osseous lesions on the jaws, that of benign fibrous-osseous lesions is generally good; such benign fibrous-osseous lesions can be completely healed with accurate diagnosis and treatment. Benign fibrous-osseous lesions clinically have various bone disorder and similar histopathological features. Such lesions are common in the head and neck region, especially in the jaws. Among these lesions, osseous dysplasia is classified as a single disease, but juvenile active ossifying fibroma and aggressive, active psammomatoid ossifying/cementifying fibroma are still debatable ${ }^{9-12}$. The various classification systems of fibrous-osseous lesions on the jaws have been introduced, but Waldron's classification ${ }^{13}$ reported in 1985 is still used. This clinical study on benign fibrous-osseous lesions classified the lesions more simply according to Waldron's modified classification ${ }^{14}$ as fibrous dysplasia, ossifying fibroma, periapical cemental dysplasia and osteoblastoma, and cementoblastoma and considered each disease's clinical aspect and radiological and histological findings.

Fibrous dysplasia is benign, a slowly growing disease with normal bone being replaced by fibrous tissue with varying calcification. The etiology is unclear yet. Houston's summarized report ${ }^{15}$ on the presented pathophysiology included 1) transformed activity of bone-forming mesenchyme, 2) expression of hyperphosphatamia-induced chronic hyperparathyroidism, 3) expression of complex metabolic disorders, 4) metabolic disorders of calcium and phosphorus, 5) non-specific reaction of bone to trauma, 6) increased secretion of estrogen, 7) congenital mesenchymal disorder, 8) form of lipoid granulomatosis, and 9) transformation of von Recklinghausen's neurofibromatosis. Nonetheless, fibrous dysplasia is accepted to be neither familial nor hereditary disease. Recently, pathologists regard it as nonneoplastic developmental disorder of bone ${ }^{16,17}$. Fibrous dysplasia is classified as monostotic and polyostotic. Polyostotic fibrous dysplasia may show metabolic disorder (i.e., Albright's syndrome). Polyostotic fibrous dysplasia may also be classified into the case related to endocrine abnormalities 
and the case without endocrine abnormalities. Polyostotic fibrous dysplasia is rarer than monostotic fibrous dysplasia, making up 30\% of total fibrous dysplasia with about $20 \%$ afflicting the mandible ${ }^{18,19}$. In this study, one case was proven to be Polyostotic fibrous dysplasia. The diseases in such case occurred simultaneously in various regions of facial bone. In whole body bone scan using radioactive isotope, There were lesions in the cervical region, thoracic vertebrae, lumbar region, unilateral scapula, and femur region as well as facial bone. The patient with such polyostotic fibrous dysplasia was a woman in her twenties, and there was no other functional disorders in the endocrine system. Four of 5 cases of fibrous dysplasia were diagnosed as monostotic fibrous dysplasia. The onset age of the patients was generally 30 s. The duration of disease was from 3 years to 10 years. According to the literature, the symptoms reported in fibrous dysplasia varied depending on the morbidity. The most dominant symptom in monostotic fibrous dysplasia in maxillofacial region is facial asymmetry caused by slow-growing mass. The main clinical symptom of patients in this study was facial asymmetry or buccal swelling, which, sometimes in the case of a small lesion, was also discovered in a routine dental exam by chance. Various radiological findings on fibrous dysplasia were reported. Radiologically, the features were classified into 3 categories such as pagetoid type, sclerotic type, and cyst-like type. In this study, the features could be mostly pagetoid type in the radiography, showing groundglass radiopacity. The lesion affected mandible ( 2 cases) and maxilla and adjacent bone regions ( 3 cases) with one case of afflicting the maxilla, zygoma, and sphenoid bones. Therefore, to find out the morbidity of such lesions, various radiographic examinations and bone scans are performed such as conventional CT and 3-dimensional CT as well as panoramic radiograph. The common histological finding on such lesions is that normal bone is replaced by cellular fibers including various minerals. At this time, there is no significant difference between the monostotic and the polyostotic type, but there is a difference primarily in the total change of fibrous dysplasia and the appearance of osteoid feature. Fibroblasts may be arranged in various forms from helical arrangement to fine fibrous stroma; a fibrous tissue contains well-differentiated cells and solid collagen matrix. Fib stroma contains irregularly arranged bony trabeculae in immature bone which is called "Chinese character trabeculae" due to the similarity with the Chinese script ${ }^{17}$. In these cases, such immature, irregular bony trabeculae distinctively contained in fibrous stroma were observed as the finding of a lump having similar form as Chinese characters, and such bony trabecular form in microscopy were considered to have diagnostic value. An indication of surgical treatment depends on an aspect of each symptom. For the lesion without symptom, surgical treatment may not be an indication. Fibrous dysplasia is generally small and slow-growing. As the lesion becomes bigger, there may be serious facial deformity and general facial abnormalities. In this study, the patients were mostly in their 30s. And there was a case of a 15-year-old girl afflicted by the disease. She came to the hospital with chief complaint of facial asymmetry since the mandible, maxilla, and zygoma were affected. Bony recontouring was performed to correct the facial deformity even though she was young. There are many surgical methods available for fibrous dysplasia. Some clinicians strongly assert that all injured bone tissues should be removed. These are based on the following theories: 1) operational therapy with incomplete excision of the lesions may accelerate the growth of the remaining lesions, 2) continuous growth of the lesions occurs in $20 \%$ of the cases, and 3) patient's burden of the remaining lesions. On the other hand, other surgeons raised doubts on the complete excision of the lesions by presenting problems in aesthetic defect after surgery or complicated reconstructive surgery. They maintained that conservative surgery including partial excision or osseous resection has a high rate of recurrence (23\%), but additional treatments are not required. Consequently, most of the patients can be fully healed through the bone regeneration process ${ }^{20}$. In this study, the examination period after the surgery was about 3 years. Since facial asymmetry in the maxilla was recognized in 2 years after surgery in one case with polyostotic fibrous dysplasia, the lesion was additionally resected. Two years later, satisfactory progress was noted.

Ossifying fibroma and cementifying fibroma are regarded as a single lesion in fibrous-osseous entity. There is only a difference in histological findings on bone between calcified element and cementum; all the other features are interchangeable with each other. Ossifying fibroma is observed mostly in the lower molar and premolar regions but are sometimes detected in the anterior maxilla and mandible as well. The lesions are slow-growing and asymptomatic ${ }^{21}$. In 6 cases of this clinical study, 2 of them were in 10s, 1 in 20s, 2 in their 40s and 1 in 50s. Most of the patients except for one were female. Lesion was in the mandibular body. Patients' chief complaints were facial swelling and pain. Our cases mostly showed the aspect of bony enlargement in buccal and lingual cortical bone and the mixed aspect of radiolucency 
with clear sclerotic bone margin with radiopacity. In the CT scan, most of the lesions showed spherical contour, and the margins were radiolucent. The histological findings on these clinical cases revealed little clear difference of lesions from adjacent normal bone tissue as the aspect of immature bony trabeculae spread into a fibrous stroma and a mixed aspect of substance showing cementum-like calcification with bony trabeculae in cases with advanced ossification. As treatment for the clinical 6 cases, limited excision of the lesion was mostly performed; no recurrence was observed. But, In one case, since wide ossifying fibroma was in the mandibular body, complaints of facial asymmetry and pain followed. Partial bone resection of the mandibular body was performed, and iliac bone grafting was carried out immediately.

Juvenile ossifying fibroma is a type of ossifying fibroma afflicting children; it has a different aspect from the one occurring among adults ${ }^{10}$. The lesion occurring in the orbital region should be separated from the one originating in the periodontal ligament. In this clinical study, one case- an adult woman in her 40s- had ossified lesion with a type of calcification similar to the one observed in juvenile ossifying fibroma. For juvenile ossifying fibroma, conservative surgical treatment is recommended, because patients with conservative surgery showed similar result with wide surgery.

Florid osseous dysplasia is a chronic, progressive disease. There are clear clinical, radiological, and histological findings on it, and the lesion is observed in each quadrant of facial bone. Florid osseous dysplasia mainly occurs in the mandibular premolar and molar region, and some patients complain of pain, tenderness, and swelling. Florid osseous dysplasia occur in both dentulous and edentulous patients. Moreover, the disease generally affects both sides of the mandibular ramus. Each of such lesions has a different size, and they can be radiologically classified into the early, middle, and late stages in terms of progress $^{22}$. Unlike general fibrous-osseous lesions, it has its distinct clinical symptom, and it may be observed in the maxilla. In this clinical study, one patient, a male in his/her 60s, came to the hospital with complaint of pain on both posterior mandible. The lesion showed the mixed radiopacity and radiolucency. At first examination, the diagnosis was fibrous dysplasia. Unlike other fibrousosseous lesions, the patient complained of continuous pain and the lesion was removed and healed uneventfully without recurrence. The histological findings finally confirmed it as florid osseous dysplasia.

Periapical cemental dysplasia, focal cemento-osseous dysplasia is generally asymptomatic, mainly occurring on the lower part of the posterior mandible unlike cementoma that occurs on the lower anterior, Radiologically, it shows radiolucency in the early stage but a mixed aspect with radiopacity in the middle stage and radiopacity with spherical shape in the late mature stage ${ }^{23}$. In these cases, the lesion affected the entire posterior mandible, and there was no difference in age and sex. Excision was done and the finally diagnosed as periapical cemental dysplasia with fibrous stroma containing immature bony trabeculae.

Osteoblastoma mainly occurs in long bone and rarely occurs maxillofacial lesions. Cementoblastoma similar to osteoblastoma occurs mainly among males rather than females. The initial symptoms are swelling and pain, and most of the patients are reported in their 10s, and facial bone lesion seems to be related to a permanent tooth and to be fused with tooth; predilection sites are the mandibular premolar and molar area ${ }^{24-27}$. Osteoblastoma seemed to be one of a bone destructive disease and most of the lesions have osteolytic borders together with the expansion of the cortical bone plate. One case was observed in our study. A 21-year-old female had lesions in the posterior mandible and came to the hospital with chief complaints of pain and swelling persisting for several months. The small spherical mandibular body lesions were mixed with radiolucency and radiopacity in the panoramic radiography, and the MRI findings revealed multiple septa in the lesions with bone expansion on the buccal side. The histological findings on osteoblastoma showed that the lesions are similar to fibrousosseous lesions, but may be mistaken for osteosarcoma due to increased cell activity. Despite a lump with calcified tissue, the characteristic findings revealed that it is a stroma with high cell differentiation and was found to be attached to the affected teeth. In our case, since the result of two biopsies before the surgery cast doubt on osteosarcoma, wide excision was performed. Iliac bone graft was done after the surgery, with the patient showing no evidence of recurrence for 2 year follow-up check. However, permanent specimen showed an interconnected form of bony trabeculae in the fibrovascular stroma, and osteoid which was diagnosed as osteoblastoma surrounded with osteoblast cells. The treatment method is to excise the relevant bone and tooth with margins by regarding it as a locally aggressive benign tumor ${ }^{26,27}$.

Fibrous-osseous lesions have similar features each other, ${ }^{9,13,16}$. However, such similarity induces difficulties in differential diagnosis. It is crucial to isolate malignant lesions with benign ones. Genetic examination is tried to diagnose and prognose the fibrous-osseous disease in advance, but clinical 
application is yet difficult. The clinical, histological, and radiological findings proven in this clinical study could be helpful as a reference indicator during treatment.

\section{Conclusion}

1. A total of 9 patients were diagnosed as malignant fibrous-osseous lesions, 8 of which had osteosarcoma and 1 with Ewing's sarcoma. Patients with osteosarcoma came to the hospital with chief complaints of swelling and tooth mobility. The radiological findings were irregular resorption of bone margins and periosteal reaction. The histological observation showed cellular pleomorphism, atypical mitosis, and separation of osteoid tissue by cellular fibrous stroma. Patients with Ewing's sarcoma had tooth mobility and swelling as chief complaints, showing onion-skin appearance in the radiography. Irregular bone expansion and radiolucency were also observed. The histological findings showed a syncytial appearance with poorly defined cell outline and cytoplasmic glycogen and vimentin-positive tumor.

2. A total of 16 patients were diagnosed as benign fibrousosseous lesions, 6 of which had fibrous dysplasia, 6 with ossifying fibroma, 3 with cemental dysplasia, and 1 with osteoblastoma. Patients with fibrous dysplasia complained of facial swelling and facial asymmetry and showed pagetoid type with ground-glass radiopacity mostly in the radiography. Histologically, an aspect of Chinese character type bony trabeculae inherent in fibrous stroma were observed. Patients with ossifying fibroma had swelling and pain. The radiological findings were radiolucency with sclerotic bone margins showing buccal and lingual cortical bone expansion, with the histological findings revealing the aspect of immature bony trabeculae spreading into a fibrous stroma. Patients with cemental dysplasia showed the limited radiolucent lesion in the bone marrow of the mandible with the well-defined border in the radiography. The histological findings showed a calcified substance similar to cement in a fibrous stroma. The chief complaints of patients with osteoblastoma were pain and swelling. Radiological findings were radiopacity showing osteolytic borders. The histological findings showed an interconnected form of bony trabeculae with osteoid in the fibrovascular stroma and osteoblasts.

Primarily, it is important to understand the difference of benign and malignant lesions and relation to a systemic disease, and proper treatment should be taken accordingly. Since a patient with benign fibrous-osseous disease is prone to recurrence and malignant transformation, close follow-up for a certain period is deemed necessary.

\section{References}

1. von Recklinghausen F. Die fibrose oderdeformiende osteite. Berlin: Festschrift R Virchow zu seinem; 1891.

2. Waldron CA. Fibro-osseous lesions of the jaws. J Oral Maxillofac Surg 1985;43:249-62.

3. Eversole LR. Clinical outline of oral pathology: diagnosis and treatment. 3rd ed. Ontario: BC Decker Inc; 1992.

4. Brannon RB, Fowler CB. Benign fibro-osseous lesions: a review of current concepts. Adv Anat Pathol 2001;8:126-43.

5. Shafer WG, Hine MK, Levy BM. A textbook of oral pathology. 4th ed. Philadelphia: WB Saunders Company; 1983.

6. Fernandes R, Nikitakis NG, Pazoki A, Ord RA. Osteogenic sarcoma of the jaw: a 10-year experience. J Oral Maxillofac Surg 2007;65:1286-91.

7. Ogunlewe MO, Ajayi OF, Adeyemo WL, Ladeinde AL, James O. Osteogenic sarcoma of the jaw bones: a single institution experience over a 21-year period. Oral Surg Oral Med Oral Pathol Oral Radiol Endod 2006;101:76-81.

8. Pitak-Arnnop P, Bellefqih S, Bertolus C, Chaine A, Dhanuthai K, Gruffaz F, et al. Ewing's sarcoma of jaw bones in adult patients: 10year experiences in a Paris university hospital. J Craniomaxillofac Surg 2008;36:450-5.

9. Eversole LR, Leider AS, Nelson K. Ossifying fibroma: a clinicopathologic study of sixty-four cases. Oral Surg Oral Med Oral Pathol 1985;60:505-11.

10. Slootweg PJ, Panders AK, Koopmans R, Nikkels PG. Juvenile ossifying fibroma. An analysis of 33 cases with emphasis on histopathological aspects. J Oral Pathol Med 1994;23:385-8.

11. Melrose RJ, Abrams AM, Mills BG. Florid osseous dysplasia. A clinical-pathologic study of thirty-four cases. Oral Surg Oral Med Oral Pathol 1976;41:62-82.

12. Regezi JA, Sciubba JJ. Oral pathology: clinical-pathologic correlations. 2nd ed. Philadelphia: WB Saunders Company; 1993: 143.

13. Waldron CA, Giansanti JS. Benign fibro-osseous lesions of the jaws: a clinical-radiologic-histologic review of sixty-five cases. II. Benign fibro-osseous lesions of periodontal ligament origin. Oral Surg Oral Med Oral Pathol 1973;35:340-50.

14. Waldron CA. Fibro-osseous lesions of the jaws. J Oral Maxillofac Surg 1993;51:828-35.

15. Houston WO Jr. Fibrous dysplasia of maxilla and mandible: clinicopathologic study and comparison of facial bone lesions with lesions affecting general skeleton. J Oral Surg 1965;23:17-39.

16. Boysen ME, Olving JH, Vatne K, Koppang HS. Fibro-osseous lesions of the cranio-facial bones. J Laryngol Otol 1979;93:793807.

17. Hamner JE 3rd, Scofield HH, Cornyn J. Benign fibro-osseous jaw lesions of periodontal membrane origin. An analysis of 249 cases. Cancer 1968;22:861-78.

18. Albright F, Butler AM, Hampton AO, Smith P. Syndrome characterized by osteitis fibrosa disseminata, areas of pigmentation and endocrine dysfunction, with precocious puberty in females. N Engl J Med 1937;216:727-46.

19. Cohen MM Jr, Howell RE. Etiology of fibrous dysplasia and McCune-Albright syndrome. Int J Oral Maxillofac Surg 1999;28:366-71.

20. Zimmerman DC, Dahlin DC, Stafne EC. Fibrous dysplasia of the maxilla and mandible. Oral Surg Oral Med Oral Pathol 1958;11:5568. 
21. Su L, Weathers DR, Waldron CA. Distinguishing features of focal cemento-osseous dysplasias and cemento-ossifying fibromas: I. A pathologic spectrum of 316 cases. Oral Surg Oral Med Oral Pathol Oral Radiol Endod 1997;84:301-9.

22. Loh FC, Yeo JF. Florid osseous dysplasia in Orientals. Oral Surg Oral Med Oral Pathol 1989;68:748-53.

23. Higuchi Y, Nakamura N, Tashiro H. Clinicopathologic study of cemento-osseous dysplasia producing cysts of the mandible. Report of four cases. Oral Surg Oral Med Oral Pathol 1988;65:339-42.

24. Störkel S, Wagner W, Makek MS. Psammous desmo-osteoblastoma. Ultrastructural and immunohistochemical evidence for an osteogenic histogenesis. Virchows Arch A Pathol Anat Histopathol 1987;411:561-8

25. Miller AS, Rambo HM, Bowser MW, Gross M. Benign osteoblastoma of the jaws: report of three cases. J Oral Surg 1980;38:694-7.

26. Dorfman HD, Weiss SW. Borderline osteoblastic tumors: problems in the differential diagnosis of aggressive osteoblastoma and lowgrade osteosarcoma. Semin Diagn Pathol 1984;1:215-34.

27. Eisenbud L, Kahn LB, Friedman E. Benign osteoblastoma of the mandible: fifteen year follow-up showing spontaneous regression after biopsy. J Oral Maxillofac Surg 1987;45:53-7. 